\title{
Increased mortality in patients with corticosteroid-dependent asthma: a nationwide population-based study
}

\author{
Hyun Lee ${ }^{1}$, Jiin Ryu², Eunwoo Nam², Sung Jun Chung ${ }^{1}$, Yoomi Yeo', \\ Dong Won Park ${ }^{1}$, Tai Sun Park ${ }^{1}$, Ji-Yong Moon ${ }^{1}$, Tae-Hyung Kim 1ㅜ, \\ Jang Won Sohn ${ }^{1}$, Ho Joo Yoon ${ }^{1}$ and Sang-Heon Kim (i) ${ }^{1}$
}

Affiliations: ${ }^{1}$ Dept of Internal Medicine, Hanyang University College of Medicine, Seoul, Korea. ${ }^{2}$ Biostatistical Consulting and Research Lab, Medical Research Collaborating Center, Hanyang University, Seoul, Korea.

Correspondence: Sang-Heon Kim, Dept of Internal Medicine, Hanyang University College of Medicine, 222 Wangsimni-ro, Seongdong-gu, Seoul 04763, Korea. E-mail: sangheonkimahanyang.ac.kr

@ERSpublications

Chronic use of systemic corticosteroid (CS) was associated with increased risk of mortality in patients with asthma, with a significant dose-response relationship between systemic CS use and long-term mortality http://bit.ly/2ku3ZJl

Cite this article as: Lee H, Ryu J, Nam E, et al. Increased mortality in patients with corticosteroiddependent asthma: a nationwide population-based study. Eur Respir J 2019; 54: 1900804 [https://doi.org/ 10.1183/13993003.00804-2019].

\section{ABSTRACT}

Introduction: Chronic systemic corticosteroid (CS) therapy is associated with an increased risk of mortality in patients with many chronic diseases. However, it has not been elucidated whether chronic systemic CS therapy is associated with increased mortality in patients with asthma. The aim of this study was to determine the effects of chronic systemic CS therapy on long-term mortality in adult patients with asthma.

Methods: A population-based matched cohort study of males and females aged $\geqslant 18$ years with asthma was performed using the Korean National Health Insurance Service database from 2005 to 2015. Hazard ratio (HR) with 95\% confidence interval for all-cause mortality among patients in the CS-dependent cohort (CS use $\geqslant 6$ months during baseline period) relative to those in the CS-independent cohort (CS use $<6$ months during baseline period) was evaluated.

Results: The baseline cohort included 466941 patients with asthma, of whom 8334 were CS-dependent and 458607 were CS-independent. After 1:1 matching, 8334 subjects with CS-independent asthma were identified. The HR of mortality associated with CS-dependent asthma relative to CS-independent asthma was 2.17 (95\% CI 2.04-2.31). In patients receiving low-dose CS, the HR was 1.84 (95\% CI 1.69-2.00); in patients receiving high-dose CS, the HR was 2.56 (95\% CI 2.35-2.80).

Conclusions: In this real-world, clinical practice, observational study, chronic use of systemic CS was associated with increased risk of mortality in patients with asthma, with a significant dose-response relationship between systemic CS use and long-term mortality. 


\section{Introduction}

Asthma affects $5-10 \%$ of the population and contributes to approximately 0.4 million deaths annually worldwide [1]. Although severe asthma affects only $4-10 \%$ of all asthma patients [2-4], that population has more asthma-related symptoms, increased risk of acute exacerbation and higher morbidity compared with those with nonsevere asthma [5]. Accordingly, severe asthma patients consume more healthcare resources than nonsevere asthma patients [6-9].

Severe asthma is difficult to manage and $20-60 \%$ of patients with severe asthma need regular use of a systemic corticosteroid (CS) $[10,11]$. Despite the beneficial effects of systemic CS [12], chronic use in asthma patients is associated with many serious complications [8, 13-17].

Chronic systemic CS use is associated with an increased risk of mortality in patients with many chronic diseases, such as rheumatoid arthritis and inflammatory bowel disease [18, 19]. However, little evidence is available on the risk of mortality associated with chronic systemic CS therapy in asthma patients [20]. In addition, it is not known whether there is a dose-dependent relationship between chronic CS use and long-term mortality in these patients.

For this study, we followed a cohort of 8334 patients with CS-dependent asthma using data from the Korean National Health Insurance Service (NHIS) to evaluate long-term mortality compared with a 1:1 matched cohort of CS-independent asthma patients. We also evaluated dose dependency in the relationship between systemic CS dose and risk of mortality.

\section{Methods}

Data source and study population

This study was approved by the Institutional Review Board of Hanyang University Hospital, Seoul, Korea (HYUH 2017-09-051). The requirement of informed consent from the participants was waived because the NHIS database was constructed after anonymisation. The NHIS provides mandatory healthcare for nearly all Korean citizens. The NHIS collects health data from nearly all 50 million of its insured subjects, including admission and outpatient visit records, diagnoses, drug prescriptions, national health examination data, and deaths. The NHIS provides all aforementioned information for research purposes [21].

From January 1, 2005 to December 31, 2005 there were 751180 asthma patients aged $\geqslant 18$ years. Of those potential participants, we excluded 1277 patients with only one visit associated with International Statistical Classification of Diseases and Related Health, 10th Revision (ICD-10) codes J45-J46 as a major or minor diagnosis. Of the remaining 749903 patients, we further excluded 50632 who had rheumatoid arthritis (M05-M06), systemic lupus erythematosus (M32) or systemic sclerosis (L94) (n=29300), inflammatory bowel disease (K50-K51) ( $\mathrm{n}=2053)$, or malignancy (C00-C99) ( $=24377)$ and those with no claims for asthma-related medications $(\mathrm{n}=232330)$. Thus, 466941 patients were included (supplementary figure E1).

\section{Definitions}

The index date was defined as 12 months from the first prescription of asthma-related medication with ICD-10 codes J45-J46 as a major or minor diagnosis between January 1, 2005 and December 31, 2005. The baseline period was defined as 12 months before the index date. The follow-up period was from the index date to the date of death or December 31, 2015, whichever was sooner (supplementary figure E2).

Asthma in adults was defined according to the following criteria: 1) age $\geqslant 18$ years, 2) at least two claims under ICD-10 codes J45-J46, and 3) at least one claim in the baseline period for prescription of asthma-related drugs such as inhaled CS (ICS) or systemic CS, bronchodilators, leukotriene receptor antagonists and xanthine derivatives [22-24]. CS-dependent asthma was defined as: 1) presence of asthma and 2) prescription of systemic CS under ICD-10 codes J45-J46 for $\geqslant 6$ months in the baseline period $[8,13,25]$. Otherwise, patients were classified as having CS-independent asthma. The proportion of patients who met the definitions of both groups during the follow-up are summarised in supplementary table E1.

Baseline comorbidities were defined as comorbidities with at least one claim under ICD-10 codes as a major diagnosis during the baseline period. New-onset comorbidities were defined as at least one claim under ICD-10 codes as a major diagnosis during follow-up but not the baseline period, as follows: diabetes mellitus (E10-E14), Cushing's syndrome (E24), adrenal insufficiency (E27.3-E27.4), bone necrosis (M87), osteoporosis (M80-M82), vertebral or pelvic bone fracture (S22.0-S22.1, S32 and M48.4), pneumonia (J12-J18), tuberculosis (A15-A19), hypertension (I10-I15), angina (I20), myocardial infarction (I21), heart failure (I50), peptic ulcer (K25-K27), gastrointestinal bleeding (K92.0-K92.2), glaucoma (H40 and H42) and cataract (H25, H26 and H28). 
To analyse causes of death, we used mortality data provided by Statistics Korea, an initiative of the Ministry of Strategy and Finance of South Korea [21]. Causes of mortality were classified under ICD-10 codes as respiratory diseases (J00-J99) including chronic lower respiratory diseases (J40-J47), cardiovascular diseases (I00-I99) including hypertension (I10-I15), ischaemic heart diseases (I20-I25), cerebrovascular diseases (I60-I69), malignant neoplasms (C00-C97), injury, poisoning and external causes (S00-T98), endocrine diseases (E00-E90) including diabetes mellitus (E10-E14), gastrointestinal diseases (K00-K93), neurological diseases (G00-G99), mental and behavioural disorders (F00-F99), musculoskeletal and connective tissue diseases (M00-M99) including osteoporosis (M80-M82), and others.

We classified CS-dependent asthma patients into high-dose and low-dose groups based on the median daily doses of systemic CS used. Low-dose systemic CS was defined as median $<5.5 \mathrm{mg}^{- \text {day }^{-1}}$ prednisolone $^{-1}$ equivalent dose and high-dose CS was defined as median $\geqslant 5.5 \mathrm{mg} \cdot$ day $^{-1}$ prednisolone equivalent dose during the baseline period.

Asthma-related emergency department (ED) visit or hospitalisation was defined as a visit to an ED or admission to a hospital under ICD-10 codes J45-J46 as a major or minor diagnosis during the follow-up period.

\section{Main outcomes and measures}

The primary outcome was all-cause mortality during the follow-up period. Secondary outcomes were healthcare use (all-cause or asthma-related ED visits or hospitalisations) and new comorbidities during the follow-up period. We additionally evaluated whether there were dose-dependent relationships between these clinical outcomes and systemic CS dose.

\section{Statistical analysis}

Baseline characteristics (age group, sex, type of insurance, Charlson Comorbidity Index (CCI) [26], comorbidities and asthma-related medications) between patients in the CS-dependent and CS-independent cohorts were compared using the McNemar test.

Controls were identified through 1:1 matching in which the nearest available neighbour for each case was selected as a control [27], i.e. within the same categories of sex and type of insurance, a control with similar age and CCI was matched for each CS-dependent asthma patient. To assess the effect of chronic CS use on the main outcomes of mortality, healthcare use (ED visits and hospitalisations), new comorbidities and their incidence rates were calculated per 100000 person-years and compared between the two patient groups by the normal approximation test for binomials.

The Kaplan-Meier method was used to estimate survival curves during the follow-up period and survival was compared among groups by the log-rank test. Hazard ratios (HRs) with 95\% confidence intervals for the main outcomes among patients in the CS-dependent cohort relative to those in the CS-independent cohort were evaluated using a Cox proportional hazards regression model. To further adjust for the effects of comorbidities on mortality, we performed a Cox proportional hazards regression model with covariate adjustment using propensity scores based on the number of comorbidities.

We also determined HRs for each cause of mortality. We used a cause-specific and subdistribution proportional hazards regression model to account for competing risks caused by mortality from other causes [28].

All analyses were conducted using SAS version 9.4 (SAS Institute, Cary, NC, USA). All tests were two-sided and p-values $<0.05$ were considered statistically significant.

\section{Results}

Population

The baseline cohort comprised 466941 patients with asthma, of whom 8334 (1.8\%) were CS-dependent and 458607 were CS-independent (supplementary figure E1). After matching, the study cohort included 8334 subjects with CS-dependent asthma matched with 8334 subjects with CS-independent asthma. As shown in table 1 , the baseline characteristics between the two cohorts were well balanced with regard to age, sex, type of insurance and CCI. However, compared with the CS-independent cohort, more patients in the CS-dependent cohort had the following comorbidities $(\mathrm{p}<0.001$ for each comorbidity comparison except for angina): diabetes mellitus, Cushing's syndrome, adrenal insufficiency, osteoporosis, vertebral or pelvic bone fracture, pneumonia, tuberculosis, angina $(\mathrm{p}=0.033)$ and congestive heart failure. Regarding asthma-related medications, the proportions of patients who received ICS (74.8\% versus 55.1\%; $\mathrm{p}<0.001$ ) and long-acting $\beta_{2}$-agonist (LABA) $(65.1 \%$ versus $43.2 \%$; $<0.001)$ were higher in the CS-dependent cohort than in the CS-independent cohort. 
TABLE 1 Descriptive characteristics of the study population

\begin{tabular}{|c|c|c|c|c|}
\hline & Total & $\begin{array}{l}\text { CS-dependent } \\
\text { asthma }\end{array}$ & $\begin{array}{c}\text { CS-independent } \\
\text { asthma }\end{array}$ & $\mathrm{p}$-value \\
\hline Subjects & 16668 & 8334 & 8334 & \\
\hline Age years & $66.0 \pm 12.5$ & $66.0 \pm 12.5$ & $66.0 \pm 12.5$ & 0.545 \\
\hline \multicolumn{5}{|l|}{ Age group } \\
\hline $20-29$ years & $144(0.9)$ & $72(0.9)$ & $72(0.9)$ & 1.0 \\
\hline $30-39$ years & $482(2.9)$ & $241(2.9)$ & $241(2.9)$ & \\
\hline 40-49 years & $1161(7.0)$ & $580(7.0)$ & $581(7.0)$ & \\
\hline $50-59$ years & $2602(15.6)$ & $1301(15.6)$ & $1301(15.6)$ & \\
\hline $60-69$ years & $4934(29.6)$ & $2467(29.6)$ & $2467(29.6)$ & \\
\hline$\geqslant 70$ years & 7345 (44.1) & $3673(44.1)$ & $3672(44.1)$ & \\
\hline Sex & & & & 1.0 \\
\hline Male & $9386(56.3)$ & $4693(56.3)$ & $4693(56.3)$ & \\
\hline Female & $7282(43.7)$ & $3641(43.7)$ & 3641 (43.7) & \\
\hline Type of insurance & & & & 1.0 \\
\hline $\begin{array}{l}\text { Self-employed health } \\
\text { insurance }\end{array}$ & $6770(40.6)$ & $3385(40.6)$ & $3385(40.6)$ & \\
\hline Employee health insurance & $9090(54.5)$ & 4545 (54.5) & $4545(54.5)$ & \\
\hline Medical aid & $808(4.9)$ & 404 (4.9) & 404 (4.9) & \\
\hline Charlson Comorbidity Index & $3.75 \pm 2.3$ & $3.75 \pm 2.3$ & $3.75 \pm 2.3$ & 1.0 \\
\hline \multicolumn{5}{|l|}{ Comorbidities } \\
\hline Metabolic disease & $5104(30.6)$ & 2709 (32.5) & 2395 (28.7) & $<0.001$ \\
\hline Diabetes mellitus & $4953(29.7)$ & $2580(31.0)$ & $2373(28.5)$ & $<0.001$ \\
\hline Cushing's syndrome & $264(1.6)$ & $232(2.8)$ & $32(0.4)$ & $<0.001$ \\
\hline Adrenal insufficiency & $164(1.0)$ & $142(1.7)$ & $22(0.3)$ & $<0.001$ \\
\hline Bone disease & $2877(17.3)$ & $1717(20.6)$ & $1160(13.9)$ & $<0.001$ \\
\hline Bone necrosis & $63(0.4)$ & $39(0.5)$ & $24(0.3)$ & 0.060 \\
\hline Osteoporosis & $2632(15.8)$ & $1574(18.9)$ & $1058(12.7)$ & $<0.001$ \\
\hline $\begin{array}{l}\text { Vertebral or pelvic bone } \\
\text { fracture }\end{array}$ & $628(3.8)$ & 406 (4.9) & $222(2.7)$ & $<0.001$ \\
\hline Infectious disease & $4833(29.0)$ & 2743 (32.9) & $2090(25.1)$ & $<0.001$ \\
\hline Pneumonia & 4469 (26.8) & $2558(30.7)$ & $1911(22.9)$ & $<0.001$ \\
\hline Tuberculosis & $1051(6.3)$ & $613(7.4)$ & $438(5.3)$ & $<0.001$ \\
\hline Cardiovascular disease & 9811 (58.9) & 4981 (59.8) & $4830(58.0)$ & $<0.001$ \\
\hline Hypertension & 9110 (54.7) & $4586(55.0)$ & 4524 (54.3) & 0.096 \\
\hline Angina & $2064(12.4)$ & 1075 (12.9) & $989(11.9)$ & 0.033 \\
\hline Myocardial infarction & $463(2.8)$ & $242(2.9)$ & $221(2.7)$ & 0.311 \\
\hline Congestive heart failure & $1767(10.6)$ & $1008(12.1)$ & $759(9.1)$ & $<0.001$ \\
\hline Gastrointestinal disease & 7075 (42.5) & $3572(42.9)$ & $3503(42.0)$ & 0.202 \\
\hline Peptic ulcer disease & 7032 (42.2) & $3547(42.6)$ & 3485 (41.8) & 0.252 \\
\hline Gastrointestinal bleeding & $165(1.0)$ & $79(1.0)$ & $86(1.0)$ & 0.574 \\
\hline Ophthalmological disease & $740(4.4)$ & $380(4.6)$ & $360(4.3)$ & 0.442 \\
\hline Glaucoma & $103(0.6)$ & $55(0.7)$ & $48(0.6)$ & 0.490 \\
\hline Cataract & $661(4.0)$ & $337(4.0)$ & 324 (3.9) & 0.599 \\
\hline \multicolumn{5}{|l|}{ Medication during follow-up\# } \\
\hline Any ICS & $10831(65.0)$ & $6236(74.8)$ & $4595(55.1)$ & $<0.001$ \\
\hline Any LABA & 9030 (54.2) & $5429(65.1)$ & $3601(43.2)$ & $<0.001$ \\
\hline SABA & 11965 (71.8) & $6801(81.6)$ & $5164(62.0)$ & $<0.001$ \\
\hline LTRA & $9401(56.4)$ & $4952(59.4)$ & $4449(53.4)$ & $<0.001$ \\
\hline
\end{tabular}

Data are presented as $n$, mean \pm SD or $n(\%)$, unless otherwise stated. CS: corticosteroid; ICS: inhaled corticosteroid; LABA: long-acting $\beta_{2}$-agonist; SABA: short-acting $\beta_{2}$-agonist; LTRA: leukotriene receptor antagonist. ${ }^{\#}$ : some patients received more than one medication.

\section{Mortality}

The overall mortality was 5191 per 100000 person-years during a median of 9.5 years (interquartile range 5.5-9.9 years) of follow-up. All-cause mortality was higher in the CS-dependent cohort than in the CS-independent cohort (6760 versus 3833 per 100000 person-years; $\mathrm{p}<0.001$ ) (figure 1a), which is consistent with our survival analysis $(\mathrm{p}<0.001$ for log-rank test) (figure 2 ). Patients in the CS-dependent cohort were 2.17 (95\% CI 2.04-2.31) times more likely to die during the follow-up period than those in the CS-independent cohort (table 2). Regardless of sex (male in supplementary figure E3 and female in supplementary figure E4) 

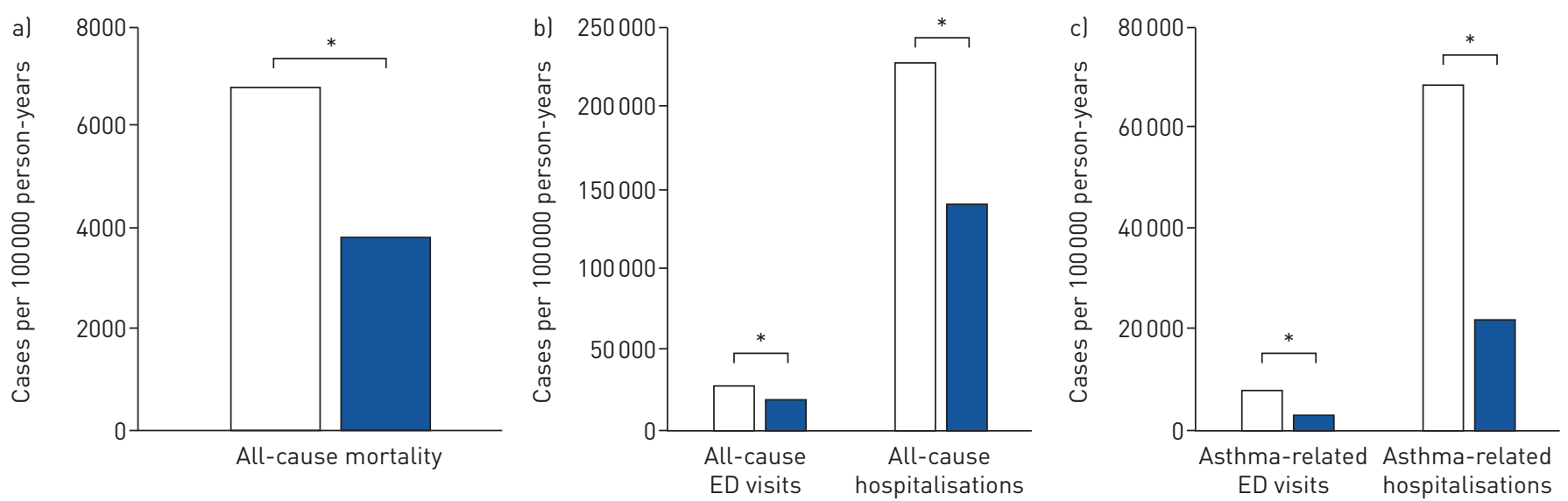

FIGURE 1 Comparison of all-cause mortality, emergency department (ED) visits and hospitalisations during follow-up. a) All-cause mortality. b) All-cause ED visits and hospitalisations. c) Asthma-related ED visits and hospitalisations. CS: corticosteroid. *: $p<0.001$.

and age group (age $<65$ years in supplementary figure E5 and age $\geqslant 65$ years in supplementary figure E6), there was a significant difference in survival between the two groups $(p<0.001$ for each sex and age group comparison). The HR of mortality in the CS-dependent cohort relative to the CS-independent cohort remained significant after adjustment for the number of comorbidities (HR 2.10, 95\% CI 1.97-2.23).

As shown in figure 3 , the survival analysis found significant differences by systemic CS dose $(\mathrm{p}<0.001)$ in the proportion of subjects who survived. In a dose-dependent manner, those who received low-dose and high-dose CS had 1.84 (95\% CI 1.69-2.00) and 2.56 (95\% CI 2.35-2.80) times higher mortality rates, respectively, than patients in the CS-independent cohort (table 2). Survival analyses performed using three categories representing CS doses $(<5,5-10$ and $\geqslant 10 \mathrm{mg}$ prednisolone equivalent) also showed significant differences in the proportion of subjects who survived (supplementary figure E7).

The common causes of mortality in asthma patients were respiratory diseases $(36.3 \%)$, cardiovascular diseases (19.3\%), malignant neoplasms (16.6\%), injury, poisoning and external causes (5.1\%), and endocrine diseases (3.4\%) (table 3). The mortality risks associated with respiratory diseases (HR 3.12, 95\% CI 2.85-3.42), cardiovascular diseases (HR 1.28, 95\% CI 1.15-1.43), malignant neoplasms (HR 1.14, 95\% CI 1.01-1.28), injury, poisoning and external causes (HR 1.40, 95\% CI 1.13-1.74), and endocrine diseases

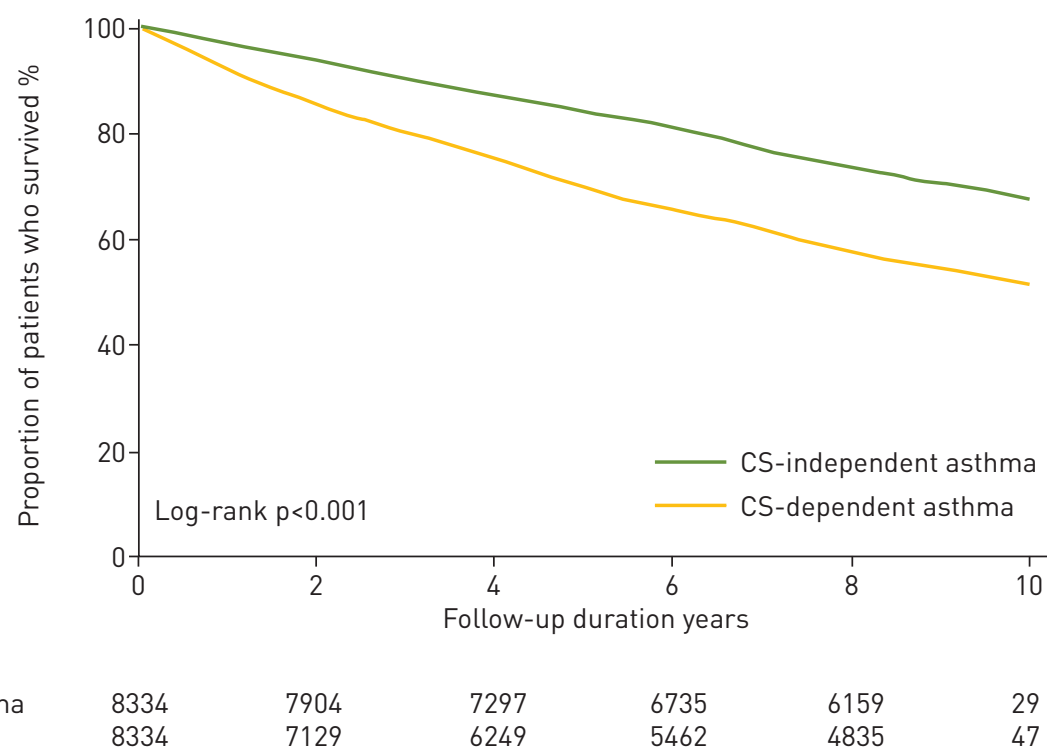

At risk $n$ :

CS-independent asthma

$8334 \quad 7129$

6249

5462

FIGURE 2 Kaplan-Meier survival analysis of time to death. CS: corticosteroid. 
TABLE 2 Effects of systemic corticosteroid (CS) dose on overall mortality, emergency department (ED) visits and hospitalisations

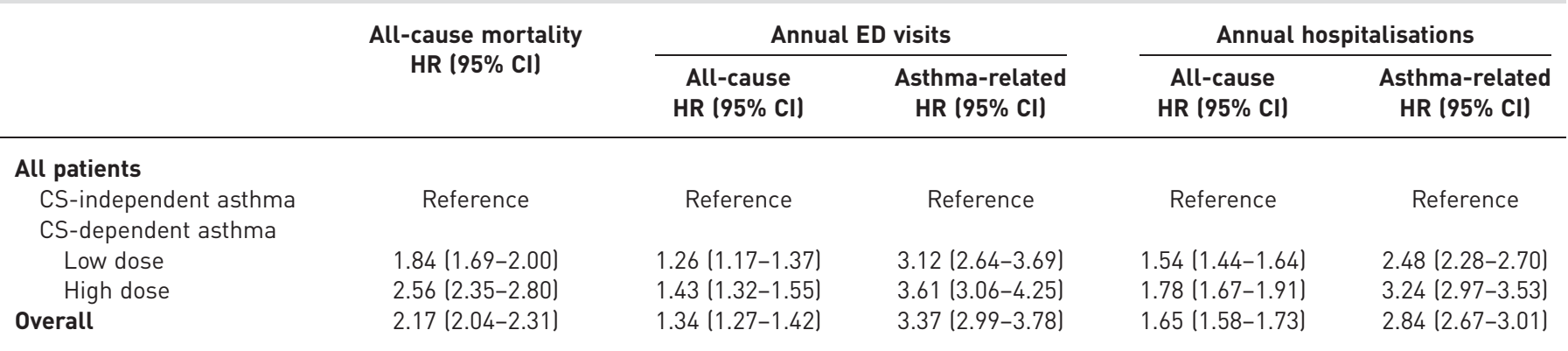

HR: hazard ratio.

(HR 1.71, 95\% CI 1.30-2.23) were higher in the CS-dependent cohort than in the CS-independent cohort. When considering competing risks caused by mortality due to other diseases, mortality risks were especially significant for respiratory diseases (subdistribution HR 2.96, 95\% CI 2.70-3.24) and endocrine diseases (subdistribution HR 1.49, 95\% CI 1.14-1.95).

\section{Healthcare use}

As shown in figure 1, the rates of annual ED visits (27429 versus 18794 per 100000 person-years; $\mathrm{p}<0.001$; figure $1 \mathrm{~b}$ ) including asthma-related ED visits (8077 versus 2681 per 100000 person-years; $\mathrm{p}<0.001$; figure 1c) and annual hospitalisations (228719 versus 140755 per 100000 person-years; $\mathrm{p}<0.001$; figure 1b) including asthma-related hospitalisations (68454 versus 21837 per 100000 person-years; $\mathrm{p}<0.001$; figure 1c) were higher in the CS-dependent cohort than in the CS-independent cohort. The HRs of ED visits and hospitalisations showed a tendency to increase as the systemic CS dose increased (table 2).

\section{Occurrence of new comorbidities}

The occurrence of new comorbidities is summarised in supplementary table E2. As shown in supplementary figure E8, compared with the patients in the CS-independent cohort, those in the CS-dependent cohort were more likely to have the following comorbidities: diabetes mellitus, Cushing's syndrome, adrenal insufficiency, bone necrosis, osteoporosis, vertebral or pelvic bone fracture, pneumonia, tuberculosis, hypertension, myocardial infarction, congestive heart failure, gastrointestinal bleeding and cataract. The HRs of all those comorbidities except for adrenal insufficiency and cataract showed a tendency to increase as the systemic CS dose increased.

At risk $n$ :

CS-independent asthma CS-dependent asthma (LD)

CS-dependent asthma (HD)

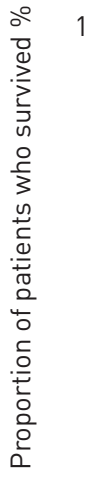

FIGURE 3 Kaplan-Meier survival analysis of time to death according to systemic corticosteroid (CS) dose. LD: low dose; HD: high dose. 
TABLE 3 Hazard ratios (HRs) for mortality in the corticosteroid (CS)-dependent cohort relative to the CS-independent cohort

\begin{tabular}{|c|c|c|c|c|c|}
\hline & \multicolumn{3}{|c|}{ Mortality rate } & \multirow{2}{*}{$\begin{array}{l}\text { Cause-specific } \\
\text { HR }(95 \% \mathrm{CI})\end{array}$} & \multirow{2}{*}{$\begin{array}{l}\text { Cause-specific } \\
\text { subdistribution } \\
\operatorname{HR}^{\text {II }}(95 \% \text { CI) }\end{array}$} \\
\hline & Total $^{\#}$ & $\begin{array}{l}\text { CS-dependent } \\
\text { cohort }\end{array}$ & $\begin{array}{c}\text { CS-independent } \\
\text { cohort }\end{array}$ & & \\
\hline Respiratory diseases & 2379 (36.3) & $1740 / 2379(73.1)$ & $639 / 2379(26.9)$ & $3.12(2.85-3.42)$ & $2.96(2.70-3.24)$ \\
\hline Chronic lower respiratory diseases & $1928(29.4)$ & $1467 / 1928(76.1)$ & $461 / 1928(23.9)$ & $3.63(3.27-4.03)$ & $3.41(3.07-3.78)$ \\
\hline Asthma & $553(8.4)$ & $437 / 553(79.0)$ & $116 / 553(21.0)$ & $4.27(3.48-5.24)$ & $3.83(3.13-4.71)$ \\
\hline Cardiovascular diseases & 1266 (19.3) & $664 / 1266(52.4)$ & $602 / 1266(47.6)$ & $1.28(1.15-1.43)$ & $1.11(0.99-1.23)$ \\
\hline Hypertension & $139(2.1)$ & $73 / 139$ (52.5) & $66 / 139(47.5)$ & $1.28(0.92-1.79)$ & $1.10(0.79-1.53)$ \\
\hline Ischaemic heart disease & $365(5.6)$ & $196 / 365(53.7)$ & $169 / 365(46.3)$ & $1.33(1.08-1.63)$ & $1.16(0.94-1.42)$ \\
\hline Heart failure & $137(2.1)$ & $77 / 137(56.2)$ & $60 / 137(43.8)$ & $1.49(1.06-2.08)$ & $1.27(0.91-1.78)$ \\
\hline Cerebrovascular disease & $411(6.3)$ & $199 / 411(48.4)$ & $212 / 411(51.6)$ & $1.09(0.90-1.33)$ & $0.94(0.77-1.14)$ \\
\hline Malignant neoplasms & $1084(16.6)$ & $535 / 1084(49.4)$ & $549 / 1084(50.6)$ & $1.14(1.01-1.28)$ & $0.97(0.86-1.09)$ \\
\hline Endocrine diseases & $221(3.4)$ & $132 / 221(59.7)$ & $89 / 221(40.3)$ & $1.71(1.30-2.23)$ & $1.49(1.14-1.95)$ \\
\hline Diabetes mellitus & $206(3.1)$ & $123 / 206(59.7)$ & $83 / 206(40.3)$ & $1.71(1.29-2.25)$ & $1.49(1.12-1.96)$ \\
\hline Gastrointestinal diseases & $146(2.2)$ & $75 / 146(51.4)$ & $71 / 146(48.6)$ & $1.24(0.90-1.72)$ & $1.06(0.76-1.46)$ \\
\hline Neurological diseases & $119(1.8)$ & $48 / 119$ (40.3) & $71 / 119(59.7)$ & $0.79(0.55-1.14)$ & $0.66(0.46-0.95)$ \\
\hline Mental and behavioural disorders & $59(0.9)$ & $31 / 59(52.5)$ & $28 / 59(47.5)$ & $1.31(0.78-2.18)$ & $1.10(0.66-1.84)$ \\
\hline $\begin{array}{l}\text { Musculoskeletal and connective } \\
\text { tissue diseases }\end{array}$ & $38(0.6)$ & $22 / 38(57.9)$ & $16 / 38(42.1)$ & $1.59(0.84-3.03)$ & $1.37(0.72-2.62)$ \\
\hline Osteoporosis & $20(0.3)$ & $11 / 20(55.0)$ & $9 / 20(45.0)$ & $1.40(0.58-3.39)$ & $1.22(0.51-2.95)$ \\
\hline Others & $904(13.8)$ & $529 / 904(58.5)$ & $375 / 904(41.5)$ & $1.63(1.43-1.86)$ & $1.42(1.25-1.62)$ \\
\hline
\end{tabular}

\section{Discussion}

Using a large-scale, population-based, longitudinal cohort study, we demonstrated that long-term use of systemic CS correlates with increased risk of mortality, ED visits, hospitalisations and development of new comorbidities in asthma patients in a dose-dependent manner.

With the development of new drugs, great improvements have been made in the treatment of patients with severe asthma, especially the type 2 phenotype [29]. However, a substantial proportion of patients are still CS-dependent [30] and, despite some improvement [12], these patients often suffer from adverse events associated with CS [8, 13-17, 31]. Although higher mortality is expected in patients with CS-dependent asthma and mortality is one of the most important issues in these patients, few longitudinal data addressing their long-term mortality are available [32]. Recently, BouRdin et al. [20] evaluated the long-term treatment outcomes of 52 patients with CS-dependent asthma and found that half of them died over 20 years. It is also noteworthy that $>60 \%$ of these patients died of fatal asthma, indicating the importance of mortality data in that population [20]. Extending those findings with a very large population-based longitudinal study, our study shows that about half of the patients died over 10 years, which is significantly higher than the percentage of CS-independent patients who died during the same period.

Another important finding of our study is that systemic CS use in asthma is associated with a dose-dependent increase in the mortality rate. A dose-dependent relationship between systemic CS and the mortality rate was demonstrated in subjects with rheumatoid arthritis [33]. On the contrary, the use of CS-sparing drugs such as methotrexate decreased mortality in patients with rheumatoid arthritis [19]. In a similar fashion, CS-sparing therapy using anti-tumour necrosis factor- $\alpha$-directed therapy in Crohn's disease reduced mortality [34]. These findings suggest that CS-sparing therapies using new biologics could have survival benefits for patients with severe asthma. Fortunately, recent studies showed that new biologics can significantly reduce severe acute exacerbations, providing systemic CS-sparing effects for 
patients with severe asthma [29]. These promising outcomes will hopefully improve mortality in patients with CS-dependent asthma.

The major causes of mortality in patients with CS-dependent asthma were respiratory diseases, cardiovascular diseases, malignant neoplasms, injury, poisoning and external causes, and endocrine diseases. Compared with patients in the CS-independent cohort, those in the CS-dependent cohort were at higher risk for death due to the aforementioned diseases, with the highest risk of mortality associated with respiratory diseases (e.g. asthma, chronic obstructive pulmonary disease and pneumonia). When we considered competing risks caused by other causes of death, risks due to respiratory diseases and endocrine diseases were especially significant, suggesting that improved treatment strategies for those diseases are urgently needed to effectively reduce mortality associated with chronic CS use.

As shown in previous studies [6-9], chronic systemic CS use is associated with the occurrence of new comorbidities and healthcare use. We carefully suggest that the occurrence of these comorbidities might have contributed to the higher mortality of patients in the CS-dependent asthma cohort. In addition, a recent study showed that having four or more CS prescriptions is significantly associated with subsequent acute exacerbation of asthma [17]. In line with that result, our study showed that asthma-related ED visits and hospitalisations were significantly higher among patients with CS-dependent asthma than among those with CS-independent asthma. It is likely that the high exacerbation rate might also have been associated with the increased mortality we found in patients with CS-dependent asthma.

Whether systemic CS and doses thereof are appropriate should be discussed. Surprisingly, $\sim 25 \%$ of CS-dependent patients did not use ICS or ICS/LABA, which suggests that systemic CS was inappropriately prescribed in Korea. There are a number of possible explanations for this phenomenon. First, the proportion of very old patients in our study was high. In real-world contexts, nonadherence regarding ICS use is an important problem in elderly patients [35]. Second, low prescription rates of ICS have been documented in real-world practice, especially in primary clinics in Korea [22-24]. Third, some physicians may believe that ICS is not necessary when a patient receives systemic CS, which is more potent than ICS. However, regardless of the reasons, our results suggest that efforts to reduce systemic CS use and enhance ICS use are urgently needed to improve the treatment outcomes of CS-dependent asthma patients. CS doses are also an important issue. In our study, we observed a dose-dependent association between CS dose and unfavourable outcomes. Thus, CS-sparing strategies administering minimal doses of systemic CS are recommended.

This study has several limitations. First, it was performed in a single country and the clinical characteristics of patients with CS-dependent asthma might vary by country or ethnicity. For example, patients with CS-dependent asthma in this study were relatively older than those in a previous study [10]. However, regardless of the age group, the mortality in the CS-dependent group was significantly higher than that in the CS-independent group. Second, no current definition of chronic asthma has been validated using electronic health records [36]. Many previous studies defined chronic asthma using the US Healthcare Effectiveness Data and Information Set (HEDIS) criteria, which includes assessments for any of the following asthma-related events over periods of 12 and 24 months: 1) ED visits, 2) hospitalisations, 3) outpatient visits and two asthma prescriptions or 4) four asthma prescriptions [36]. Since data associated with ED visits and hospitalisations are also included as claims data in the NHIS database, the definition of chronic asthma used in this study is very similar to that recognised by HEDIS criteria. In addition, we used the same definition that was used in several previous studies evaluating asthma in Korea using the NHIS database [22-24]. Thus, despite some limitations, the definition of asthma used in this study is acceptable in the current situation, as an internationally accepted definition of chronic asthma is not available. Third, the proportion of patients who met the definition of CS-dependent asthma gradually decreased during follow-up, which might have attenuated the accumulating effects of systemic CS use. Despite this limitation, our results clearly show that even chronic CS use over a certain time period may be associated with very unfavourable treatment outcomes. In addition, using a definition of CS-dependent asthma that has been used in previous studies [8, 13, 25] allowed us to identify a stable control group; $>97 \%$ of the patients who were classified as CS-independent remained CS-independent during follow-up. The stable control group also underscores the reliability of our observations of higher mortality in our CS-dependent asthma cohort.

In summary, in this population-based retrospective cohort study, chronic systemic CS use was associated with a higher rate of mortality in a dose-dependent manner in asthma patients. CS-sparing treatment strategies are urgently needed to improve the treatment outcomes of patients with CS-dependent asthma.

Author contributions: Literature search: all authors; study design: H. Lee, J. Ryu, E. Nam, J-Y. Moon, H.J. Yoon and S-H. Kim; data analysis: H. Lee, J. Ryu, E. Nam and S-H. Kim; data interpretation: all authors; writing: H. Lee, J. Ryu, E. Nam and S-H. Kim; tables and figures: H. Lee, J. Ryu and S-H. Kim. 
Conflict of interest: None declared.

Support statement: This study was funded by the Korea Ministry of Environment (MOE) as the Environmental Health Action Program (2016001360003) and a grant of the Korea Health Technology R\&D Project through the Korea Health Industry Development Institute (KHIDI), funded by the Ministry of Health and Welfare, Korea (HI19C0218). The Korea MOE and KHIDI played no role in the design of the study, analysis and interpretation of data, and writing the current manuscript. Funding information for this article has been deposited with the Crossref Funder Registry.

\section{References}

1 GBD 2015 Chronic Respiratory Disease Collaborators. Global, regional, and national deaths, prevalence, disability-adjusted life years, and years lived with disability for chronic obstructive pulmonary disease and asthma, 1990-2015: a systematic analysis for the Global Burden of Disease Study 2015. Lancet Respir Med 2017; 5: 691-706.

2 Chung KF, Wenzel SE, Brozek JL, et al. International ERS/ATS guidelines on definition, evaluation and treatment of severe asthma. Eur Respir J 2014; 43: 343-373.

3 Hekking PP, Wener RR, Amelink M, et al. The prevalence of severe refractory asthma. J Allergy Clin Immunol 2015; 135: 896-902.

4 Larsson K, Stallberg B, Lisspers K, et al. Prevalence and management of severe asthma in primary care: an observational cohort study in Sweden (PACEHR). Respir Res 2018; 19: 12.

5 McDonald VM, Gibson PG. Exacerbations of severe asthma. Clin Exp Allergy 2012; 42: 670-677.

6 Sadatsafavi M, Lynd L, Marra C, et al. Direct health care costs associated with asthma in British Columbia. Can Respir J 2010; 17: 74-80.

7 Sullivan PW, Campbell JD, Ghushchyan VH, et al. Characterizing the severe asthma population in the United States: claims-based analysis of three treatment cohorts in the year prior to treatment escalation. J Asthma 2015; 52: 669-680.

8 Lefebvre P, Duh MS, Lafeuille MH, et al. Burden of systemic glucocorticoid-related complications in severe asthma. Curr Med Res Opin 2017; 33: 57-65.

9 Voorham J, Xu X, Price DB, et al. Healthcare resource utilization and costs associated with incremental systemic corticosteroid exposure in asthma. Allergy 2019; 74: 273-283.

10 Heaney LG, Brightling CE, Menzies-Gow A, et al. Refractory asthma in the UK: cross-sectional findings from a UK multicentre registry. Thorax 2010; 65: 787-794.

11 Kim M-H, Kim S-H, Park S-Y, et al. Characteristics of adult severe refractory asthma in Korea analyzed from the severe asthma registry. Allergy Asthma Immunol Res 2019; 11: 43-54.

12 Phipatanakul W, Mauger DT, Sorkness RL, et al. Effects of age and disease severity on systemic corticosteroid responses in asthma. Am J Respir Crit Care Med 2017; 195: 1439-1448.

13 Lefebvre P, Duh MS, Lafeuille MH, et al. Acute and chronic systemic corticosteroid-related complications in patients with severe asthma. J Allergy Clin Immunol 2015; 136: 1488-1495.

14 Dalal AA, Duh MS, Gozalo L, et al. Dose-response relationship between long-term systemic corticosteroid use and related complications in patients with severe asthma. J Manag Care Spec Pharm 2016; 22: 833-847.

15 Bloechliger M, Reinau D, Spoendlin J, et al. Adverse events profile of oral corticosteroids among asthma patients in the UK: cohort study with a nested case-control analysis. Respir Res 2018; 19: 75.

16 Price DB, Trudo F, Voorham J, et al. Adverse outcomes from initiation of systemic corticosteroids for asthma: long-term observational study. J Asthma Allergy 2018; 11: 193-204.

17 Sullivan PW, Ghushchyan VH, Globe G, et al. Oral corticosteroid exposure and adverse effects in asthmatic patients. J Allergy Clin Immunol 2018; 141: 110-116.

18 Lewis JD, Gelfand JM, Troxel AB, et al. Immunosuppressant medications and mortality in inflammatory bowel disease. Am J Gastroenterol 2008; 103: 1428-1435.

19 Chester Wasko M, Dasgupta A, Ilse Sears G, et al. Prednisone use and risk of mortality in patients with rheumatoid arthritis: moderation by use of disease-modifying antirheumatic drugs. Arthritis Care Res 2016; 68: 706-710.

20 Bourdin A, Molinari N, Vachier I, et al. Mortality: a neglected outcome in OCS-treated severe asthma. Eur Respir J 2017; 50: 1701486

21 Lee J, Lee JS, Park SH, et al. Cohort profile: The National Health Insurance Service-National Sample Cohort (NHIS-NSC), South Korea. Int J Epidemiol 2017; 46: e15.

22 Choi JY, Yoon HK, Lee JH, et al. Nationwide use of inhaled corticosteroids by South Korean asthma patients: an examination of the Health Insurance Review and Service database. I Thorac Dis 2018; 10: 5405-5413.

23 Cho EY, Oh KJ, Rhee CK, et al. Comparison of clinical characteristics and management of asthma by types of health care in South Korea. J Thorac Dis 2018; 10: 3269-3276.

24 Kim S, Kim J, Kim K, et al. Healthcare use and prescription patterns associated with adult asthma in Korea: analysis of the NHI claims database. Allergy 2013; 68: 1435-1442.

25 Wysocki K, Park SY, Bleecker E, et al. Characterization of factors associated with systemic corticosteroid use in severe asthma: data from the Severe Asthma Research Program. J Allergy Clin Immunol 2014; 133: 915-918.

26 Charlson ME, Pompei P, Ales KL, et al. A new method of classifying prognostic comorbidity in longitudinal studies: development and validation. J Chronic Dis 1987; 40: 373-383.

27 Bergstralh E, Kosanke J. Computerized matching of cases to controls. Technical report 56. 1995. www.mayo.edu/ research/documents/biostat-56pdf/doc-10026923 Date last accessed: September 8, 2019.

28 Fine JP, Gray RJ. A proportional hazards model for the subdistribution of a competing risk. J Am Stat Assoc 1999; 94: 496-509.

29 McGregor MC, Krings JG, Nair P, et al. Role of biologics in asthma. Am J Respir Crit Care Med 2019; 199: 433-445.

30 Sweeney J, Brightling CE, Menzies-Gow A, et al. Clinical management and outcome of refractory asthma in the UK from the British Thoracic Society Difficult Asthma Registry. Thorax 2012; 67: 754-756.

31 Zazzali JL, Broder MS, Omachi TA, et al. Risk of corticosteroid-related adverse events in asthma patients with high oral corticosteroid use. Allergy Asthma Proc 2015; 36: 268-274. 
32 Levy ML, Winter R. Asthma deaths: what now? Thorax 2015; 70: 209-210.

33 del Rincón I, Battafarano DF, Restrepo JF, et al. Glucocorticoid dose thresholds associated with all-cause and cardiovascular mortality in rheumatoid arthritis. Arthritis Rheumatol 2014; 66: 264-272.

34 Lewis JD, Scott FI, Brensinger CM, et al. Increased mortality rates with prolonged corticosteroid therapy when compared with antitumor necrosis factor- $\alpha$-directed therapy for inflammatory bowel disease. Am J Gastroenterol 2018; 113: 405-417.

35 Gibson PG, McDonald VM, Marks GB. Asthma in older adults. Lancet 2010; 376: 803-813.

36 Al Sallakh MA, Vasileiou E, Rodgers SE, et al. Defining asthma and assessing asthma outcomes using electronic health record data: a systematic scoping review. Eur Respir J 2017; 49: 1700204. 